\title{
Study on the Correlation between Extracellular Matrix Protein-1 and the Growth, Metastasis and Angiogenesis of Laryngeal Carcinoma
}

\author{
Xin-Yu Meng, Juan Liu, Feng Lv, Ming-Qiu Liu, Jing-Ming Wan*
}

\begin{abstract}
Objective: To investigate the correlation between extracellular matrix protein-1 (ECM1) and the growth, metastasis and angiogenesis of laryngeal carcinoma. Materials and Methods: Forty-five samples with laryngeal benign and malignant tumors confirmed by pathology in Laiwu City People's Hospital from March 2006 to March 2011 were collected, in which there were 29 cases with laryngeal carcinoma and 16 with benign tumors. The expression of ECM1 and factor VIII-related antigens in patients with laryngeal carcinoma and those with benign tumors was respectively detected using immunohistochemical method, and the correlation between ECM1 staining grade and microvessel density (MVD) was analyzed. Results: In laryngeal carcinoma tissue, ECM1 was mainly expressed in cytoplasm, less in cytomembrane or intercellular substance. With abundant expression in the tissue of laryngeal benign tumors (benign mesenchymoma and hemangioma), ECM1 was primarily expressed in the connective tissue, which was different from the expression in laryngeal carcinoma tissue. The proportion of positive ECM1 staining (++) in patients with laryngeal carcinoma was dramatically higher than those with benign tumors $(p<0.05)$, and that of strongly-positive ECM1 staining $(+++)$ slightly higher. The results of Spearman nonparametric correlation analysis revealed that ECM1 staining grade in laryngeal carcinoma tissue had a significantly-positive correlation with MVD $(r=0.866, p=0.000)$. Conclusions: ECM1 expression in laryngeal carcinoma is closely associated with tumor cell growth, metastasis and angiogenesis, which can be considered as an effective predictor in the occurrence and postoperative recurrence of laryngeal carcinoma.
\end{abstract}

Keywords: Extracellular matrix protein-1 - laryngeal carcinoma - benign tumors - microvessel density

Asian Pac J Cancer Prev, 16 (6), 2313-2316

\section{Introduction}

Laryngeal carcinoma is a common malignant tumor, and its incidence is only secondary to nasopharyngeal carcinoma and paranasal sinus cancer in the field of otorhinolaryngology, approximately 30\% 40\% in head and neck cancer and $1.0 \% \sim 2.5 \%$ in human malignant tumors (Parkin et al., 1999). In recent years, the incidence of laryngeal carcinoma has been on the rise dramatically (Duzlu et al., 2015). Infiltration and metastasis of laryngeal carcinoma are the basic biological characteristics of malignant tumors and also one of the major reasons for poor prognosis of malignant tumors.

Generation of tumor cells originates from weakened expression of tumor suppressor genes or enhanced expression of oncogenes at the level of genes. Different from normal cells, the biological characteristics of tumor cells are intimately related to extracellular environment. Extracellular matrix protein-1 (ECM1), a sort of secretory glycoprotein with molecular weight of $85 \mathrm{kDa}$, binds to a variety of substances in the extracellular matrix, such as hyaluronic acid, chondroitin A, fibronectin and heparin, to participate in various pathological and physiological processes through different structural domains (Mathieu et al., 1994; Sercu et al., 2008). A lot of studies have demonstrated that ECM1 is related to tumor cell infiltration, metastasis and angiogenesis in the tumor tissue and expressed in a variety of tumor tissues, such as hepatocellular carcinoma, gastric cancer, lung cancer, colorectal cancer, breast cancer, thyroid carcinoma and laryngeal carcinoma (Chen et al., 2011; Wu et al., 2012; Wu et al., 2014). At present, there are few studies on ECM1 expression in laryngeal carcinoma tissue and its significance. Hence, in this study, the relationships between ECM1 and the growth, metastasis and angiogenesis of laryngeal carcinoma were analyzed so as to provide more clinical evidences for the diagnosis, treatment and prognosis of laryngeal carcinoma.

\section{Materials and Methods}

\section{General data}

Forty-five samples with laryngeal benign and malignant tumors confirmed by pathology in Laiwu City 
Xin-Yu Meng et al

People's Hospital from March 2006 to March 2011 were collected, in which there were 37 males and 8 females, 29 cases with laryngeal carcinoma and 16 with benign tumors. Among the patients with laryngeal carcinoma, there were 24 males and 5 females. They were at the age of $36 \sim 74$, with the mean age of $(56.9 \pm 9.5)$. Three patients suffered from lymph node metastasis and 8 from recurrence. All of them were followed up 2 3 years. According to clinical staging criteria of Union for International Cancer Control (UICC), there were 6 patients in phase I ( 2 cases encountered recurrence), 7 in phase II ( 3 cases encountered recurrence), 3 in phase III and 13 in phase IV ( 3 cases suffered from recurrence and 3 from lymph node metastasis). Among the patients with benign tumors, there were 13 males and 3 females. They were 12 56 years old, averagely (30.2 \pm 15.7$)$ years old. Most of them were diagnosed with papilloma. The samples above were cut into slices with the thickness of $4 \mu \mathrm{m}$.

\section{Methods}

Reagent: Concentrated primary antibodies (dilution with antibody diluent according to 1: 400 proportion) and secondary antibodies (dilution with phosphate buffer solution according to 1: 250 proportion) of ECM1 were provided by Shanghai Ruiqi Biological Technology Co., Ltd. Universal biotin-marked anti-goat SP-9000 kit, polyclonal antibody working solution of rabbit antihuman factor VIII-related antigens, antibody diluent, pepsin digestive juice, AEC, phosphate buffer solution and corresponding fluoromount-G were all purchased from Beijing Zhongshan Golden Bridge Bio-Tech Co., Ltd.

Immunohistochemical method of ECM1 and factor VIII-related antigens: Immunohistochemical staining of ECM1 was as follows: a. conduct the paraffin section and preparation first, and then dewax routinely till hydration after baking $30 \mathrm{~min}$ in a $60^{\circ} \mathrm{C}$ oven; b. swash with distilled water and apply phosphate buffer solution to soak for 5 min after incubating 5 min with $10 \%$ hydrogen peroxide; c. dropwise add the sealing normal goat serum working solution, incubate $15 \mathrm{~min}$ at room temperature and outwell; d. dropwise add primary antibody diluent of ECM1, stay overnight at $4^{\circ} \mathrm{C}$ and swash 5 min with phosphate buffer solution 3 times; e. dropwise add secondary antibody diluent of ECM1, incubate $1 \mathrm{~h}$ at room temperature and swash 5 min with phosphate buffer solution twice, and then swash again with distilled water; f. prepare AEC for coloration about $15 \mathrm{~min}$; g. redyeing $20 \mathrm{~s}$ with hematoxylin, then seal the sections after washing out, finally place the sections in a $70^{\circ} \mathrm{C}$ oven for desiccation. Immunohistochemical staining of factor VIIIrelated antigens was performed strictly based on the kit instructions. The known positive sections were regarded as a positive control and phosphate buffer solution as a negative control instead of primary antibodies.

\section{Judging criteria}

Positive ECM1 staining was considered as presence of brownish red staining in intracellular substance and cytoplasm. According to the degree and range, staining gradings were divided into 4 kinds, namely stronglypositive $(+++)$, positive $(++)$, weakly-positive $(+)$ and negative (-).

Judging criteria for microvessels: Presence of brownish red staining in vascular endothelial cells was considered as positive expression, and the most distinct area was confirmed after successful staining. The number of microvessels was calculated in the visual field of light microscope $(\times 200)$. Three highest numbers in the microvessel area were determined, and their average value was taken as the microvessel density (MVD) of this tissue (Yang et al., 1998).

\section{Statistical data analysis}

SPSS 17.0 statistical software was used to analyze the data statistically. Enumeration data were compared by chisquare test and expressed in the formation of percentages. The correlation between ECM1 staining gradings in laryngeal carcinoma tissue and MVD was analyzed using Spearman nonparametric correlation analysis. $\mathrm{p}<0.05$ was considered to be statistically significance.

\section{Results}

\section{ECM1 expression in laryngeal carcinoma tissue}

In laryngeal carcinoma tissue, ECM1 was mainly expressed in cytoplasm, less in cytomembrane or intercellular substance. Besides, it was also strongly expressed in some tumor cells, but no expression was found in the cytoplasm of some tumor cells, and it was only expressed in cytomembrane or intercellular substance (Figure 1). ECM1 was not only expressed in the carcinoma nests, but also between carcinoma nests. Lymph node metastasis would appear when ECM1 was expressed between carcinoma nests. In the tumor cytoplasm of metastatic lymph nodes, a large amount of ECM1 staining could be seen, and non-staining tumor cells were barely found (Figure 2).

\section{ECM1 expression in the tissue of laryngeal benign tumors}

With abundant expression in the tissue of laryngeal benign tumors (benign mesenchymoma and hemangioma), ECM1 was primarily expressed in the connective tissue, which was different from the expression in laryngeal carcinoma tissue. Its expression in papilloma was similar to laryngeal carcinoma, but it was mainly expressed in cytomembrane or intercellular substance, less in cytoplasm and sporadically distributed like gridding (Figure 3).

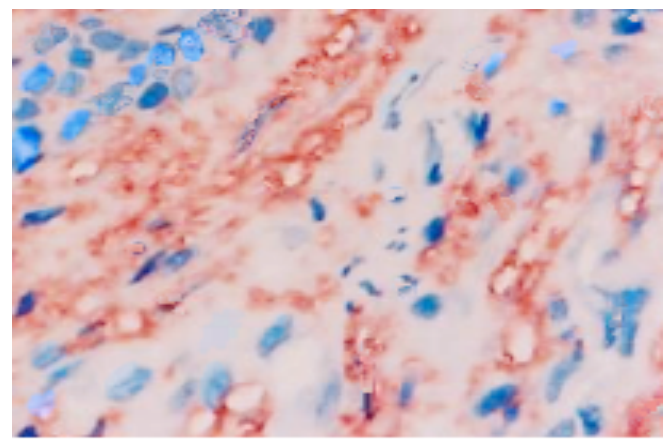

Figure 1. Positive Expression of ECM1 in Laryngeal Squamous Cell Carcinoma(SP×200) 


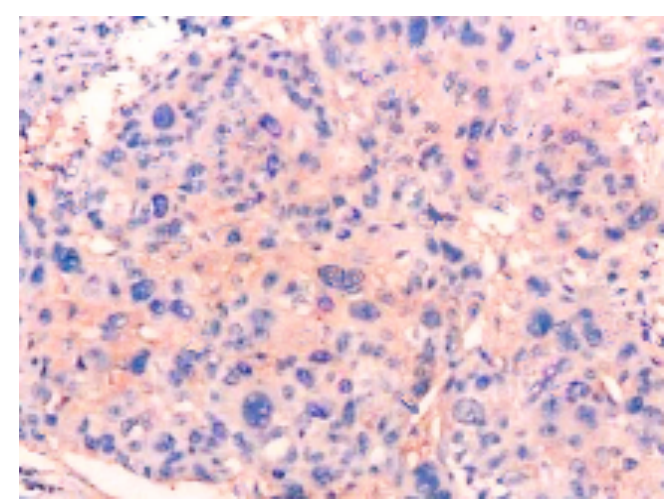

Figure 2. Positive Expression of ECM1 in Metastatic Lymph Nodes (SP×200)

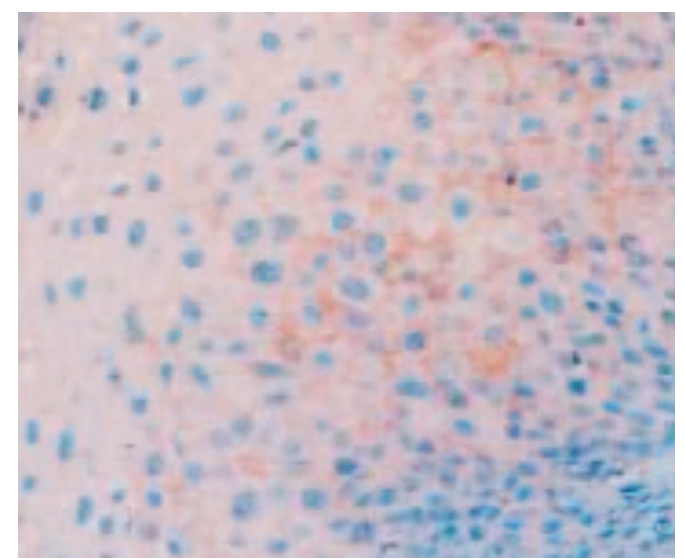

Figure 3. Positive Expression of ECM1 in Papilloma (SP×200)

Table 1. Comparison on the Stain Gradings of ECM1 in Laryngeal Carcinoma and Benign Tumors[n(\%)]

\begin{tabular}{lrrrrr}
\hline Classification & $\mathrm{n}$ & \multicolumn{1}{c}{++} & ++ & + & - \\
\hline Laryngeal carcinoma & 29 & $13(44.8)$ & $15(51.7)^{*}$ & $1(3.4)^{* *}$ & $0(0.0)$ \\
Benign tumors & 16 & $3(18.8)$ & $3(18.8)$ & $10(62.5)$ & $0(0.0)$ \\
Total cases & 45 & $16(35.6)$ & $18(40.0)$ & $11(24.4)$ & $0(0.0)$ \\
\hline
\end{tabular}

*Compared with benign tumors, ${ }^{*} \mathrm{p}<0.05$, or $* * \mathrm{p}<0.01$

Comparison on the stain gradings of ECM1 in laryngeal carcinoma and benign tumors

The proportion of positive ECM1 staining (++) in patients with laryngeal carcinoma was dramatically higher than those with benign tumors $(\mathrm{p}<0.05)$, and that of strongly-positive ECM1 staining (+++) slightly higher than those with benign tumors. The proportion of weaklypositive ECM1 staining (+) in patients with laryngeal carcinoma was distinctly lower than those with benign tumors $(\mathrm{p}<0.01)$ (Table 1).

Correlation between ECM1 stain gradings in laryngeal carcinoma tissue and MVD

The results of Spearman nonparametric correlation analysis revealed that ECM1 stain gradings in laryngeal carcinoma tissue had a significantly-positive correlation with MVD $(24.51 \pm 5.61)(\mathrm{r}=0.866, \mathrm{p}=0.000)$.

\section{Discussion}

Laryngeal carcinoma is the third common malignant tumor in the head and neck, with the major pathological type being squamous cell carcinoma, approximately accounting for $96 \%$ 98\%. According to some statistics, the incidence of laryngeal carcinoma is the highest in the north of China, especially in the northeast. It approximately occupies $7.9 \% \sim 35 \%$ in otorhinolaryngeal carcinoma (Dong et al., 2013; Meng et al., 2013). At present, the pathogenic and progressive mechanisms of laryngeal carcinoma are still not illustrated clearly. The study demonstrated that the mutual action of multiple factors can induce laryngeal carcinoma, in which apoptosis and abnormal proliferation caused by modulation of proto-oncogenes is one of the important mechanisms for occurrence of laryngeal carcinoma (Markou et al., 2013; Kum et al., 2014).

Different from normal cells, tumor cells are not constrained by peripheral tissue and have the biological characteristics of continuous proliferation, infiltration into peripheral tissue, distant metastasis, etc. A key step of tumor infiltration is the presence of heterogeneous adhesion of tumor cells and extracellular matrix, and extracellular matrix is degraded. As a secretory glycoprotein, ECM1 that was separated from osteogenic cell line MN7 in mice first could have the capabilities of translocator or binding growth and differentiation factors. It was found that ECM1 was highly expressed in a variety of tumors like breast cancer and hepatocellular carcinoma, and was closely related to tumor invasion and metastasis. Additionally, the positive rate of ECM1 expression in metastatic tumors was higher than that in non-metastatic tumors (Lee et al., 2014; Lal et al., 2009; Hou et al., 2004). In-vitro studies revealed that ECM1 could decrease the activity of matrix metalloproteinase- 9 (MMP-9) through mutual action with MMP-9 (Fujimoto et al., 2006). It could also promote the angiogenesis, invasion and metastasis of liver carcinoma via mutual action of vascular endothelial growth factor (VEGF) and MMP-9. Its abnormal expression might be considered as a predictor for poor prognosis of patients and postoperative recurrence (Chen et al., 2011). ECM1 may play an important role in the angiogenesis, and the most powerful evidence is the proliferation of human umbilical vein endothelial cells and angiogenesis of chick chorioallantoic membranes stimulated by purified recombinant ECM1.

By acting on other cells, ECM1 secreted by some tumor cells produces angioblast substances to promote angiogenesis and lymphoangiogenesis (Zhu et al., 2014). Due to a certain defects of new vessels (A large gap between endothelial cells can make cells enter into lumens easily.), the tumor cells can easily enter into blood or lymphatic vessels, consequently leading to metastasis. And meanwhile, new vessels provide the required nutriments for tumor growth. The results in this study revealed that except for papilloma, the expression of ECM1 in laryngeal benign tumors was different from laryngeal carcinoma tissue, and its expression intensity was also significantly lower than in laryngeal carcinoma tissue; ECM1 was abundantly expressed in hemangioma, mainly in connective tissue. These results further confirm that ECM1 is closely associated with angiogenesis. In papilloma, ECM1 was primarily expressed in cytomembrane or intercellular substance, 
less in cytoplasm, but its expression was on the rise in papilloma accompanied by atypical hyperplasia, indicating that ECM1 has a certain correlation with the overgrowth of cells. ECM1 was expressed in both laryngeal carcinoma and benign tumors, and the expression in laryngeal carcinoma was stronger than in benign tumors $(\mathrm{p}<0.05)$, and that in laryngeal carcinoma with lymph node metastasis stronger than without lymph node metastasis, suggesting that the intensity of ECM1 expression in laryngeal carcinoma is associated with presence or absence of lymph node metastasis. The reasons are as follows: on one hand, ECM1 can exert a stimulative effect in the occurrence and metastasis of laryngeal carcinoma; one the other hand, ECM1 is related to angiogenesis, whereas the angiogenesis effect of laryngeal carcinoma with lymph node metastasis is stronger than that without lymph node metastasis (Gu et al., 2013). In the tumor cytoplasm of metastatic lymph nodes, a large amount of ECM1 staining could be seen, and non-staining tumor cells were barely found, but no ECM1 staining was found in normal lymph nodes, showing that the metastasis occurred in the tumor cells where ECM1 was expressed. These results suggest that ECM1 expression is associated with migration of tumor cells. Additionally, the intensity of ECM1 expression in laryngeal carcinoma tissue had a significantly-positive correlation with MVD, displaying that ECM1 expression in laryngeal carcinoma tissue is related to tumor angiogenesis.

From basic experiments to clinical research, all the results display that ECM1 plays a certain role in the occurrence and progression of various malignant tumors. As a secretory glycoprotein, the content of serum ECM1 can predict tumorigenesis and postoperative recurrence. As a signal peptide secreted by tumor cells, it can promote tumor cell growth, metastasis and angiogenesis. The results in this study hint that ECM1 expression in laryngeal carcinoma is closely associated with tumor cell growth, metastasis and angiogenesis, which can be considered as an effective predictor in the occurrence and postoperative recurrence of laryngeal carcinoma.

\section{References}

Chen H, Jia WD, Li JS, et al (2011). Extracellular matrix protein 1 , a novel prognostic factor, is associated with metastatic potential of hepatocellular carcinoma. Med Oncol, 1,318-25.

Dong JR, Guo N, Zhao JP, et al (2013). Inhibition of nemo-like kinase increases taxol sensitivity in laryngeal cancer. Asian Pac J Cancer Prev, 14, 7137-41.

Duzlu M, Karamert R, Tutar H, et al (2015). Neutrophillymphocyte ratio findings and larynx carcinoma: a preliminary study in Turkey. Asian Pac J Cancer Prev, 16, 351-4.

Fujimoto N, Terlizzi J, Aho S, et al (2006). Extracellular matrix protein 1 inhibits the activity of matrix metalloproteinase 9 through high-affinity protein/proteininteractions. Exp Dermatol, 15, 300-7.

Gu M, Guan J, Zhao L, et al (2013). Correlation of ECM1 expression level with the pathogenesis and metastasis of laryngeal carcinoma. Int J Clin Exp Pathol, 6, 1132-7.

Hou YQ, Fan LY, Tu XQ, et al (2004). Expression of extracellular matrix protein 1 in tumors and its significance. Academic $J$ Second Military Medical University, 25, 1314-6.
Lee KM, Nam K, Oh S, et al (2014). Extracellular matrix protein 1 regulates cell proliferation and trastuzumab resistance through activation of epidermal growth factor-signaling. Breast Cancer Res, 16, 479.

Kum RO, Ozcan M, Baklaci D,et al (2014). Elevated neutrophilto-lymphocyte ratio in squamous cell carcinoma of larynx compared to benign and precancerous laryngeal lesions. Asian Pac J Cancer Prev, 15, 7351-5.

Lal G, Hashimi S, Smith BJ, et al (2009). Extracellular matrix 1 (ECM1) expression is a novel prognostic marker for poor long-term survival in breast cancer: a Hospital-based Cohort Study in Iowa. Ann Surg Oncol, 16, 2280-7.

Mathieu E, Meheus L, Raymackers J, et al (1994). Characterization of the osteogenic stromal cell line MN7: identification of secreted MN7 proteins using twodimensional polyacrylamide gel electrophoresis, western blotting, and microsequencing. J Bone Miner Res, 9, 903-13.

Markou K, Christoforidou A, Karasmanis I, et al (2013). Laryngeal cancer: epidemiological data from Northern Greece and review of the literature. Hippokratia, 17, 313-8.

Meng FS, Gao S, Xu Q, et al (2013). Study on the expression of Caspase-3 protein in laryngeal squamous cell carcinoma and its comparative relationship with cell apoptosis of laryngeal carcinoma. J Clin Experimental Medicine, 12, 258-60.

Parkin DM, Pisani P, Ferlay J (1999). Estimates of the worldwide incidence of 25 major cancers in 1990. Int J Cancer, 80, 827-41.

Sercu S, Zhang L, Merregaert J (2008). The extracellular matrix protein 1: its molecular interaction and implication in tumor progression. Cancer Invest, 26, 375-84.

Wu Q, Li X, Yang H, et al (2014). Extracellular matrix protein 1 is correlated to carcinogenesis and lymphatic metastasis of human gastric cancer. World J Surg Oncol, 12, 132.

Wu QW, She HQ, Liang J, et al (2012). Expression and clinical significance of extracellular matrix protein 1 and vascular endothelial growth factor-C in lymphatic metastasis of human breast cancer. BMC Cancer, 12, 47.

Yang A, Kaghad M, Wang Y, et al (1998). p63, a p53 homolog at 3q27-29, encodes multiple products with transactivating, death-inducing, and dominant-negative activities. Mol Cell, 2, 305-16.

Zhu XJ, Liu J, Xu XY, et al (2014). Novel tumor-suppressor gene epidermal growth factor-containing fibulin-like extracellular matrix protein 1 is epigenetically silenced and associated with invasion and metastasis in human gastric cancer. $\mathrm{Mol}$ Med Rep, 9, 2283-92. 